\title{
Adrenocortical Cancer: A 20-Year Experience of a Single Referral Center in Prognosis and Outcomes
}

Authors

Christos Parianos ${ }^{1,2}$, Georgios Kyriakopoulos ${ }^{1,3}$, Ioannis D. Kostakis ${ }^{4}$, Narjes Nasiri-Ansari ${ }^{\mathbb{D}}$, Chrysanthi Aggeli², Anastasia Dimitriadi ${ }^{5}$, Anna Angelousi ${ }^{6}$, Athanasios G. Papavassiliou ${ }^{1}$, Gregory A. Kaltsas ${ }^{7}$, George Zografos $^{2 *}$, Eva Kassi ${ }^{1,7^{*}}$

Affiliations

1 Department of Biological Chemistry, National and Kapodistrian University of Athens, Athens, Greece

2 Department of Surgery, General Hospital of Athens "G. Gennimatas", Athens, Greece

3 Department of Pathology, Evaggelismos Hospital, Athens, Greece

4 Department of Hepato-Pancreato-Biliary Surgery and Liver Transplantation, Royal Free Hospital, London, UK

5 Department of Pathology, General Hospital of Athens " $G$. Gennimatas", Athens, Greece

6 Unit of Endocrinology, First Department of Internal Medicine, Laiko Hospital, National and Kapodistrian University of Athens, Athens, Greece

7 Unit of Endocrinology, First Department of Propaedeutic Internal Medicine, Laiko Hospital, National \& Kapodistrian University of Athens, Athens, Greece

Key words

ACC, melan A, p53, Weiss score, Helsinki score

received 19.04.2021

accepted after revision $\quad 01.10 .2021$

Bibliography

Horm Metab Res 2021; 53: 709-716

DOI 10.1055/a-1666-9026

ISSN 0018-5043

(c) 2021. Thieme. All rights reserved.

Georg Thieme Verlag, Rüdigerstraße 14,

70469 Stuttgart, Germany

Correspondence

Eva Kassi

Department of Biological Chemistry

Unit of Endocrinology

School of Medicine

National and Kapodistrian University of Athens

75 MikrasAsias Straße

Goudi TK

11527 Athens

Greece

Tel.: + 30 2107462699; + 306974856280 ,

evakassis@gmail.com
丹 Supplementary material is available under https://doi.org/10.1055/a-1666-9026

\section{ABSTRACT}

Adrenocortical carcinoma (ACC) is a rare but very aggressive endocrine malignancy with poor survival. Histopathology is important for diagnosis, while in some cases immunohistochemical markers and gene profiling of the resected tumor may be superior to current staging systems to determine prognosis. We aimed to present the 20-year experience at a tertiary hospital in patients with ACCs and correlate the immunohistochemical characteristics of ACCs with the clinical and morphological characteristics of the tumors and the survival of the patients. Forty-five patients with ACC were included in the study. All the resections were R0. The tumor size and weight, the disease stage (ENSAT classification), Weiss score and Helsinki score were examined along with immunohistochemical expression of inhibin-A, melan A, calretinin, Ki67, synaptophysin, p53, vimentin, CKAE1/AE3. The male to female ratio was $1: 1.37$. The median age at diagnosis was 55.5 years (IQR 19-77). The median size of ACCs was $9 \mathrm{~cm}$ (IQR $3.5-22 \mathrm{~cm}$ ) and the median weight $127 \mathrm{~g}$ (IQR $18-1400 \mathrm{~g})$. The median follow up period was 18 months (IQR 1-96). Ki67 varied from $<1 \%$ to $75 \%$ (median: $16.4 \%$ ). The expression of melan-A and lower expression of Ki-67 $(\leq 4)$ were independently associated with longer OS time ( $p=0.01$ and $p=0.04$, respectively). In multivariable analysis, tumor volume $>400 \mathrm{~cm}^{3}(p=0.046)$, Weiss score $>5(p=0.007)$ and overexpression of $p 53(p=0.036)$ were independent risk factors for shorter survival. Adrenocortical carcinoma is a rare and very aggressive endocrine malignancy. The most important factors that determine long-term prognosis of ACC are the disease stage at diagnosis, the Weiss score, and the Ki67 index. Immunohistochemical markers such as melan A could also serve as prognostic factors. 


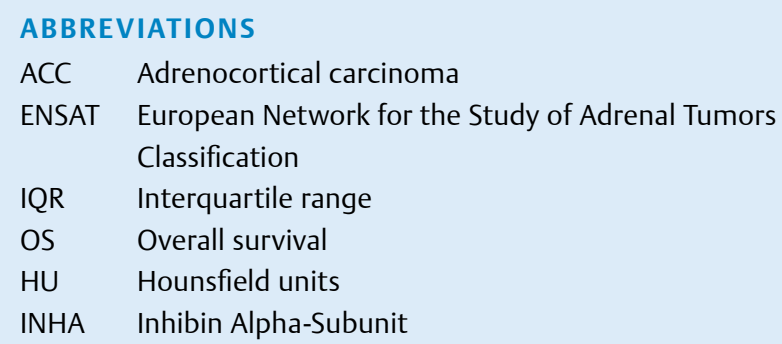

\section{Introduction}

Adrenocortical carcinoma (ACC) is a malignant neoplasm of adrenocortical origin. It affects 1-2 patients per million individuals per year and accounts for $0.02-0.2 \%$ of all cancer deaths. It tends to be slightly more common in women [1-3]. ACC can occur at any age, but there is a relatively higher incidence in children $<5$ years of age and adults in their fourth to fifth decade of life. The long-term outcome is poor with a 5-year survival rate of $60-80 \%$ for ACC localized to the adrenal gland, 35-50\% for ACC with locally advanced disease and $0-28 \%$ for metastatic ACC, indicating the prognostic relevance of tumor staging [4]. ACC incidence is 10-fold higher in Southern Brazil children, where a specific TP53 germline mutation $(\mathrm{R} 337 \mathrm{H})$ has been detected. Although most ACCs are sporadic, $<10 \%$ of the cases can occur as part of hereditary syndromes including Li-Fraumeni syndrome (TP53 mutations), BeckwithWiedemann syndrome (IGF-II overexpression), and familial adenomatous polyposis coli (APC and CTNNB1 genes) [5].

Clinical presentation of ACC is variable. Many ACCs are biochemically functional and are associated with glucocorticoid excess (Cushing's syndrome), sex steroid hypersecretion (virilizing or rarely feminizing signs), combined glucocorticoid and sex steroid excess, or mineralocorticoid overproduction [6]. However, a significant proportion of these tumors may be unassociated with syndromes of steroid excess and typically present with abdominal or flank pain due to a large retroperitoneal mass [7, 8]. There are also cases where clinical manifestations are totally absent, and the tumor is discovered incidentally or even metastatic at time of presentation [9].

The diagnosis of ACC is not always easy since there are cases that are difficult to be distinguished from benign adenomas and from tumors of other origin such as pheochromocytoma, liposarcoma or malignant melanoma [10]. SF1 remains a valid marker to document the adrenal origin with a high sensitivity and specificity, while if it is not available, others markers including inhibin-A, melan A, and calretinin can be useful when combined [11]. Weiss score, based on a combination of 9 histological criteria remains the best validated score to distinguish adenomas from ACCs. A Weiss score $>3$ and a Helsinki score $>8.5$ are strongly suggestive of malignancy $[12,13]$. A Weiss score $>3$ and recently a Ki67 labeling index $>10 \%$ are strongly suggestive of malignancy. Of interest, other biomarkers have recently emerged, amongst them proteins involved in cell proliferation and mitotic spindle regulation, beta-catenin pathway, DNA damage repair, telomere regulation and PI3K signaling pathway, with IGF-2 being the most promising. Indeed, juxtanuclear Golgi pattern of IGF-2 expression along with al- tered reticulin framework and angioinvasion were found to be the most useful diagnostic markers of ACC [14].

The most important clinical factors that determine long-term prognosis of ACC are the disease stage at presentation (according to ENSAT system), radical surgery and age. However, Ki67 remains the most powerful prognostic marker in both localized and advanced ACC. Of interest, proliferative activity/tumor grade and cortisol excess have also been recognized as independent prognostic parameters [15].

Surgery includes complete margin-negative resection of the tumor with the adrenal gland and when necessary, en bloc resection of invaded organs and peri-aortic retroperitoneal lymphadenectomy $[16,17]$. Open adrenalectomy with locoregional lymph node removal is recommended when preoperative diagnosis or high level of suspicion of ACC exists.

In the current study, we aimed to present the 20-year experience at a tertiary Hospital in patients with ACCs and correlate the immunohistochemical (IHC) features of ACCs with the clinical - morphological data and survival of patients.

\section{Ethics Approval}

This is a retrospective cohort study conducted at the Third Department of Surgery at General Hospital of Athens "G. Gennimatas". Ethical approval for this study was granted upon evaluation by the Scientific Committee of Athens General Hospital. The study was conducted according to the guidelines of the Declaration of Helsinki and its later amendments or comparable ethical standards, and it was also approved by the Institutional Ethics Committee of G. Gennimatas General Hospital of Athens, Greece (9253/17.03.2017). Written consent was obtained from all patients. A total of 45 cases of ACC were operated between June 1997 and September 2019, by the same surgeon.

\section{Patients and Methods}

The preoperative diagnosis, operative details, complications, length of hospital stay, morbidity, pathology report, and follow up were retrieved from the hospital records of 742 patients who underwent 758 adrenalectomies for benign and malignant tumors in the same period. Preoperative localization was established in all patients by computerized tomography (CT) or magnetic resonance imaging (MRI). Endocrinological evaluation and complete adrenal dynamic testing were performed to determine whether the tumor was functional or not.

The cases were reviewed and a diagnosis of ACC was confirmed using histopathological examination after surgical resection. Immunohistochemical analysis for Inhibin-A, Melan A, Calretinin, Ki67, Synaptophysin, CKAE1/AE3, p53, Vimentin has also been performed.

Demographic and clinical characteristics of the patients including also the stage of the disease, the localization, the weight and size of tumor, type of surgical procedure and the type of hypersecreted steroids, in cases of functional tumor, are provided in $>$ Table 1. All the operations, open or laparoscopic, were performed with the patient in the lateral decubitus position. Therefore, there was no need to change the position of the patient in the event of a conversion to an open operation. A transperitoneal lateral approach was 
- Table 1 Demographic, clinical, and histologic characteristics of patients with ACC.

\begin{tabular}{|c|c|c|}
\hline & Number & $\%$ \\
\hline \multicolumn{3}{|l|}{ Gender } \\
\hline women & 26 & 57.7 \\
\hline men & 19 & 42.3 \\
\hline \multicolumn{3}{|l|}{ Age } \\
\hline$<20$ & 1 & 2.2 \\
\hline $20-30$ & 2 & 4.45 \\
\hline $31-40$ & 2 & 4.45 \\
\hline $41-50$ & 14 & 31.1 \\
\hline $51-60$ & 10 & 22.2 \\
\hline $61-70$ & 8 & 17.8 \\
\hline$>70$ & 8 & 17.8 \\
\hline \multicolumn{3}{|l|}{ Tumor size (greatest dimension) } \\
\hline$<10 \mathrm{~cm}$ & 30 & 66.7 \\
\hline$\geq 10 \mathrm{~cm}$ & 15 & 33.3 \\
\hline \multicolumn{3}{|l|}{ Tumor weight } \\
\hline$<300 \mathrm{~g}$ & 31 & 68.9 \\
\hline$\geq 300 \mathrm{~g}$ & 14 & 31.1 \\
\hline \multicolumn{3}{|l|}{ Lateralization } \\
\hline Left adrenal & 26 & 57.7 \\
\hline Right adrenal & 19 & 42.3 \\
\hline \multicolumn{3}{|l|}{ Stage (ENSAT) } \\
\hline 1 & 3 & 6.6 \\
\hline II & 28 & 62.3 \\
\hline III & 12 & 26.7 \\
\hline IV & 2 & 4.4 \\
\hline \multicolumn{3}{|l|}{ Surgical approach } \\
\hline Open adrenalectomy & 30 & 66.7 \\
\hline Laparoscopic adrenalectomy & 12 & 26.7 \\
\hline Conversion to open & 3 & 6.6 \\
\hline Resection of additional organs (kidney, spleen) & 10 & 22.2 \\
\hline \multicolumn{3}{|l|}{ Hormonal activity } \\
\hline Functional & 17 & 37.7 \\
\hline Nonfunctional & 28 & 62.3 \\
\hline
\end{tabular}

used to perform laparoscopic adrenalectomy. The adrenal gland was not grasped during dissection in order to avoid troublesome bleeding or tumor disruption. The specimen in laparoscopic procedures was placed in a special bag and was extracted through minimal extension of the initial incision.

Thirty surgeries were open adrenalectomies, 12 laparoscopic and 3 other laparoscopic surgeries were converted to open surgery. Additional organs (kidney, spleen) were resected in 10 operations. There was no surgical mortality. Median hospital stay was significantly longer in the open compared to laparoscopic surgery group (median 7 compared to 3 days respectively).

Routine lymphadenectomy during surgical resection of ACC has not been performed in any of our cases. A few lymph nodes (1-5) were included in specimens where resection of additional organs was performed (peripheral pancreatectomy and splenectomy or nephrectomy ). Para-aortic lymphadenectomy is performed when indicated from imaging or intraoperative inspection. The role of lymph node dissection in the management of ACC remains controversial $[18,19]$.

\section{Histopathological evaluation and Immunohistochemical staining}

Histological diagnosis of ACC was recorded by a reference pathologist on tumor tissue removed at surgery. All cases were evaluated on a routine hematoxylin-eosin-stained slide according to the Weiss criteria in which the presence of three or more criteria highly correlates with malignant behavior [13]. The Helsinki score was determined as follows: $(5 \times$ presence of necrosis $)+(3 \times$ mitotic rate $>5$ per 50 high-power fields) + proliferation index value in the most proliferative area of tumor. The thresholds used for malignancy were a total score $\geq 3$ for the Weiss score and a total score $>8.5$ for the Helsinki score.

Immunohistochemical studies were performed on a representative deparaffinized tissue section after antigen retrieval using appropriate positive and negative controls in all cases. The Ki67 index was evaluated as a proliferation marker to assess ACC prognosis using the anti-human Ki67 antibody (clone MIB-1, DAKO). Ki-67 index was estimated as the percentage of the number of immunostained nuclei among the total number of nuclei of tumor cells (count 500-2000 tumor cells) in the high-power field measured in chosen areas of the highest density of positive nuclei (hotspots). Tumor stage was evaluated according to the classification of ACC proposed by the European Network for the Study of Adrenal Tumours (ENSAT). Immunohistochemical analysis was performed using anti p53 (clone DO-7, DAKO), CK-PAN (clone AE1/AE3, DAKO), Inhibin-A (clone EP378, BioSB), Melan A (clone A103, DAKO), Calretinin (clone DAK-Carelt1, DAKO), Synaptophysin (clone DAK-SYNAP, DAKO) and Vimentin (clone V9, DAKO) antibodies. Immunohistochemical analysis was conducted using DAKO EnVision FLEX (Dako, Carpenteria, CA, USA). The staining intensity in each slide was evaluated independently by two different pathologists, blinded to any clinical information prior to pathological review and interpretation for the purpose of this study. Immunohistochemical staining was considered positive when more than $10 \%$ of them were stained with the respective antibodies. For the evaluation of p53, the nuclear expression of p53 protein in few or in almost all tumor cells but with variable intensity was considered as normal/wild type pattern indicative of no underlying TP53 mutation. The strong nuclear expression in at least $50 \%$ of the tumor cell was defined as overexpression while the overexpression in at least $>80 \%$ was considered strongly associated with TP53 mutation [20-22].

\section{Statistical analysis}

The Shapiro-Wilk test was used for the assessment of normality of data distribution. For qualitative variables, comparisons among two or more groups were made using Chi-squared test or Fisher's exact test. For quantitative parameters, comparisons among two groups were made with Student's t-test, Welch's t-test or Mann-Whitney U-test, as appropriate, whereas comparisons among three or more 
groups were made with ANOVA or Kruskal-Wallis test, as appropriate. Correlations between two qualitative variables were tested with Pearson's correlation coefficient or Spearman's rank correlation coefficient, as indicated. Kaplan-Meier curves and the log-rank test were used for the assessment and comparison of overall survival among groups. Cox regression was performed for multivariate survival analysis. All the tests were two-tailed and the results were considered statistically significant if the $p$-value was less than 0.05 . The 25th edition of Statistical Package for Social Sciences (SPSS) (IBM Corporation, Armonk, NY, USA) was used for statistical analysis.

\section{Results}

\section{Demographic and clinical data}

Of a total of 45 patients who diagnosed with ACC the majority were female (1.37:1) with a mean age of $54.64 \pm 13.3$ years and median age of 55.5 years (IQR 19-77) at diagnosis.

All the resections were $\mathrm{R} 0$. There were 2 cases of inoperable ACC, where only biopsy of the tumor was performed, but these 2 cases were not included in our study. The surgeon systematically removed the adrenal tumor with the surrounding fat.

In all patients, routine biopsies were taken from the remnant adipose tissue in the four dimensions. To note, there was a case of laparoscopic adrenalectomy for benign disease in a patient who presented with ACC at the site of the resection, three years later. The patient underwent excision of the tumor, upper pole nephrectomy and distal pancreatectomy. The initial histology slides were reviewed and showed adenoma Weiss score II.

Tumors were located in the left adrenal gland in 26 (57.7\%) patients and in the right adrenal gland in 19 (42.3\%). In our cohort no cases of bilateral ACC, or hereditary syndrome were identified.

The main clinical symptoms were hormone related (hypercortisolism) while no other symptoms such as abdominal or flank pain and fatigue were observed. ACC was associated with hormonal overproduction in 17 patients (37.7\%) as follows: cortisol overproduction in 15 patients (33.3\%), and overproduction of more than one hormone (cortisol and androgens or estrogens) in 2 patients (4.4\%). There was no case of aldosterone overproduction.

Clinical follow up was available for 38 out of 45 patients with a median follow up period of 18 months (IQR 1-96 months). Patients were staged according to the ENSAT staging system; 3 (6.6\%) cases were classified as stage I, 28 (62.3\%) as stage II, 12 (26.7\%) as stage III and 2 (4.4\%) as stage IV ( Table 1 ). Seven patients (15.5\%) lost to follow up.

From the 12 cases of stage III by the ENSAT classification, only 2 were considered stage II preoperatively and proved to be infiltrative to surrounding tissues (stage III) during the operation. There was one recurrence in the tumor bed, treated with reoperation and one recurrence with multiple bilateral lung metastases treated conservatively. All the patients received mitotane postoperatively with the exception of two patients with ACCs smaller than $5 \mathrm{~cm}$ diameter. Furthermore, two patients with stage IV and 9 out of 12 patients with stage III received chemotherapy in addition to mitotane. Sixteen patients have died of their disease 1 to 36 months after diagnosis while the 1 -year survival was $60.5 \%$ and 5 -year survival was $18.4 \%$.

\section{Macroscopic and histopathological characteristics of the ACCs}

The characteristic gross features of malignancy (such as hemorrhage and necrosis) were detected in all cases of ACCs. Normal adrenal tissue was not distinguished in all the resected samples. The median size of ACCs was $9 \mathrm{~cm}$ (IQR 3.5-22 cm) in greatest dimension and their weight ranged from 18 to $1400 \mathrm{~g}$ (median weight of $127 \mathrm{~g}$ ). The median size of right-sided lesions was $8.5 \mathrm{~cm}$ (range: $5-22 \mathrm{~cm}$ ) and the median diameter of left-sided lesions was $8 \mathrm{~cm}$ (range: $1.5-18 \mathrm{~cm}$ ).

\section{Correlation between morphological characteristics of ACCs and Weiss score}

The ACC tumor volume and diameter was higher in right-sided lesions reaching statistical significance $(p=0.08$ and $p=0.07$, respectively). Moreover, a significant correlation between tumor volume $(p=0.011, r=0.418)$, diameter $(p=0.005, r=0.449)$, weight $(p=0.04, r=0.339)$ and Weiss score was observed.

\section{Immunohistochemical markers of ACCs}

The IHC characteristics of the 45 adrenocortical tumors are presented in Supplementary Table $1 \mathrm{~S}$. The diagnosis of ACC was confirmed histologically by Weiss score $>3$, with a range $4-8$. The mitotic count ranged from 2 to 28 mitoses per 50 high power fields. Twenty-eight of ACC tumors were surrounded by fibrous capsule while 3 of our cases showed capsular invasion (no data regarding the presence of fibrous capsule was available in 14 patients' histological reports). In our series, Ki67 varied from < $1 \%$ to $75 \%$ (median:16.4\%).

All except 5 tumors were stained positive for synaptophysin, while nine (20\%) tumors for CKAE1/AE3, 34 (75.5\%) tumors for Melan-A, 41 (91.11\%) tumors for inhibin A, 37 (82.22\%) tumors for vimentin, and $40(88.88 \%)$ tumors for calretinin were positively stained.

Statistical analysis showed that the expression of Ki-67 was positively correlated with Weiss score $(p=0.25, r=0.383)$ as it was expected. Our data also provide evidence for an association between Weiss score and the expression of vimentin and synaptophysin ( $p=0.02$ and $p=0.07$, respectively). The correlation between the expression of CKAE1/AE3 as well as Inhibin $A$ and Weiss score shows a trend towards significance ( $p=0.14$ and $p=0.14$ respectively). Moreover, the relation of CKAE1/AE3 and Inhibin A expression with Helsinki score missed statistical significance threshold ( $p=0.11$ and $p=0.13$, respectively). $\mathrm{P53}$ overexpression was related with higher Ki-67 ( $p<0.001)$ as well as with higher Helsinki score $(p<0.001)$. No statistical significance was observed regarding the correlation between IHC markers and ENSAT staging (I/II vs. III/IV).

\section{Correlation of clinical and IHC features with OS in ACC Patients}

The median follow-up was 18 months (IQR 1-96). Seven patients (15.5\%) lost to follow up, the 1 -year survival was $60.5 \%$ and 5 -year survival was $18.4 \%$. Survival was significantly higher in patients with stage I/II as compared to patients with stage III/IV ACC (62 \pm 8.7 months vs. $31 \pm 12.1$ months, $p=0.02$ ), as expected.

A larger tumor diameter $>10 \mathrm{~cm}(p=0.007)$, tumor volume $>500 \mathrm{~cm}^{3}(p=0.0003)$, tumor weight $>300 \mathrm{~g}(p=0.003)$, Ki-67 
- Table 2 Average and median of survival period in patients with ACC in relation to immunohistochemical markers.

\begin{tabular}{|c|c|c|c|c|c|c|}
\hline \multicolumn{2}{|c|}{ Immunohistochemical staining } & \multirow{2}{*}{$\begin{array}{c}\text { Average period of } \\
\text { survival (months) } \\
61.5\end{array}$} & \multirow{2}{*}{$\begin{array}{l}\text { Median Period of } \\
\text { survival (months) } \\
\mathrm{NR}^{*}\end{array}$} & \multirow{2}{*}{$\begin{array}{c}\text { Standard } \\
\text { error }\end{array}$} & \multirow{2}{*}{$\begin{array}{l}\text { 95\% Confidence } \\
\text { interval } \\
\text { 31.5-91.5 }\end{array}$} & \multirow{3}{*}{$\begin{array}{l}\text { Log Rank } \\
\text { test } \\
p=0.88\end{array}$} \\
\hline \multirow{2}{*}{ CKAE1/AE3 } & Yes $(n=9)$ & & & & & \\
\hline & No $(n=28)$ & 53.9 & 30 & 8.6 & $37.1-70.7$ & \\
\hline \multirow{2}{*}{ Inhibin A } & Yes $(n=35)$ & 56.3 & NR & 8.1 & $40.4-72.2$ & \multirow{2}{*}{$p=0.17$} \\
\hline & No $(n=3)$ & 13 & 13 & 0 & $13-13$ & \\
\hline \multirow{2}{*}{ P53 } & Yes (normal) $(n=31)$ & 57.5 & NR & 8.7 & $40.5-74.5$ & \multirow{2}{*}{$p=0.29$} \\
\hline & Overexpression $(n=7)$ & 24 & 11 & 8.5 & $7.4-40.6$ & \\
\hline \multirow{2}{*}{ Melan A } & Yes $(n=32)$ & 61.8 & NR & 8.7 & $45.2-78.4$ & \multirow{2}{*}{$p=0.01$} \\
\hline & No $(n=6)$ & 15.1 & 15 & 8.5 & $4-26.1$ & \\
\hline \multirow{2}{*}{ Synaptophysin } & Yes $(n=33)$ & 53.9 & 30 & 8.3 & $37.6-70.3$ & \multirow{2}{*}{$p=0.76$} \\
\hline & No $(n=5)$ & 60 & NR & 19.6 & $21.6-98.4$ & \\
\hline \multirow{2}{*}{ Vimentin } & Yes $(n=33)$ & 57.2 & NR & 8.2 & $41.1-73.4$ & \multirow{2}{*}{$p=0.1$} \\
\hline & No $(n=5)$ & 10.7 & 12 & 3.8 & $3.2-18.1$ & \\
\hline \multirow[b]{2}{*}{ Ki67 } & $\leq 4 \%(n=9)$ & 81 & NR & 13 & $55.5-106.5$ & \multirow[b]{2}{*}{$p=0.04$} \\
\hline & $>4 \%(n=29)$ & 40.4 & 24 & 7.9 & $24.8-55.9$ & \\
\hline
\end{tabular}

The patients lost to follow-up have been excluded from the analysis. " NR: Not reached yet.

- Table 3 Multivariable Cox-regression analysis in 45 ACC patients.

\begin{tabular}{|c|c|c|c|c|}
\hline & \multirow{2}{*}{$\begin{array}{l}\text { p- } \\
\text { Value }\end{array}$} & \multirow{2}{*}{ HR } & \multicolumn{2}{|c|}{$95.0 \% \mathrm{Cl}$ for $\operatorname{Exp}(\mathrm{B})$} \\
\hline & & & Lower & Upper \\
\hline Gender (male) & 0.599 & 0.557 & 0.063 & 4.940 \\
\hline Age $>50$ & 0.162 & 0.171 & 0.014 & 2.032 \\
\hline Stage III/IV & 0.772 & 0.703 & 0.065 & 7.650 \\
\hline Volume $>400 \mathrm{~cm}^{3}$ & 0.046 & 11.472 & 1.046 & 125.799 \\
\hline CKAE1/AE3 & 0.098 & 0.073 & 0.003 & 1.614 \\
\hline p53 overexpression & 0.036 & 21.287 & 1.214 & 373.182 \\
\hline Melan A & 0.054 & 0.103 & 0.010 & 1.040 \\
\hline Synaptophysin & 0.984 & 0.954 & 0.011 & 84.638 \\
\hline Vimentin & 0.050 & 0.019 & 0.000 & 1.003 \\
\hline Weiss score $>5$ & 0.007 & 212.502 & 4.410 & 10240.462 \\
\hline
\end{tabular}

index $>4 \%(p=0.04)$, Weiss score $>5(p=0.01)$, Helsinki score $>8$ $(p=0.06)$ were significantly associated with shorter overall survival (OS) in the univariable analysis. On the contrary, the expression of Melan A was independently associated with longer OS time $(p=0.01)$. The relation between average and median survival period and the expression of immunohistochemical markers in patients with ACC are summarized in > Table 2.

In multivariable analysis, tumor volume $>400 \mathrm{~cm}^{3}(p=0.046)$, Weiss score $>5(p=0.007)$ and overexpression of $p 53(p=0.036)$ were independent risk factors for shorter survival. In more details, Cox regression analysis showed that patients with tumor volume
( $>400 \mathrm{~cm}^{3}$ ), p53 overexpression or higher Weiss score (>5) seem to imply a higher risk of death by $11.47,21.29$ and 212.5 times, respectively ( $\triangleright$ Table 3 ).

\section{Discussion}

This study describes a single center experience in adrenocortical tumors overlooking a 20-year period. Forty-five ACCs (6\%) were diagnosed among 742 patients who underwent adrenalectomy due to adrenal masses.

ACCs are typically large tumors with diameter $>8 \mathrm{~cm}$ usually weighing more than $100 \mathrm{~g}[23,24]$. In our series, ACCs weight ranged from 18 to $1400 \mathrm{~g}$ (median weight $=127 \mathrm{~g}$ ) and the median tumor size was $9 \mathrm{~cm}$ (IQR $3.5-22 \mathrm{~cm}$ ) in greatest dimension. Tumor size is a good predictor of malignancy since tumors with diameter $>6 \mathrm{~cm}$ have shown a $25 \%$ chance of being malignant compared to $2 \%$ of those with a size $<4 \mathrm{~cm}$ [25]. However, recent studies demonstrated that ACC risk per size in adrenal incidentaloma (AI) is less than previously reported. In a retrospective review of 2219 Als, the optimal cut-off diameter for ACC was $4.6 \mathrm{~cm}$ and a combination of thorough hormonal evaluation and imaging characteristics along with the size of the tumor should better guide the management of Al [26].

According to our data, tumor volume, diameter, weight were significantly correlated with Weiss score as well as with ENSAT stage. Moreover, tumor volume $>500 \mathrm{~cm}^{3}$ was related with shorter survival rate in the multivariable analysis. In line with previous reports $[27,28]$, we found that most of the ACC cases were women at forthfifth decade of life, with stage II (ENSAT criteria) and located on the left, albeit not all studies agree with the left sided preponderance [29]. 
We also observed a trend for positive correlation between tumor size (volume and diameter) and left localization. Moreover, left ACCs had higher Helsinki score, while no correlation between tumor laterality and Weiss score, ki-67, and stage was indicated.

In line with the literature [30], we found that the mean survival of ACCs with cortisol hypersecretion was shorter (31.4 months) compared to non-secreted ACCs ( 61.5 months).

The histopathologic diagnosis of ACC is based on the recognition of several morphologic parameters none of which is in itself pathognomonic for malignancy. Moreover, there is no single distinctive immunohistochemical marker indicative of malignancy, often making it necessary to use a wider panel of parameters for confirmation of the diagnosis [20]. For this reason the Weiss score that combined morphologic and immunohistochemical criteria was introduced. A Weiss score $>3$ criteria has a specificity $>95 \%$ and a sensitivity of $100 \%$ for malignancy in many series [31].

ENSAT has shown that Ki-67 proliferation index is the most powerful prognostic marker in both localized and advanced ACC indicative of aggressive behavior and that higher Ki-67 levels are consistently associated with a worse prognosis [9]. Of note, different cut off points for Ki-67 have been suggested as predictive of overall survival and disease-free survival, by various studies [28, 32-34], which could be attributed - among others - to differences in the number of patients and in the follow-up period among studies, as well as possible inter-laboratory variations in the Ki-67 assessment in ACCs.

In our series, $\mathrm{Ki}-67$ was varied from $1 \%$ to $75 \%$ and its expression was positively correlated with Weiss score $(p=0.025, r=0.383)$ and strongly correlated with Helsinki score $(p<0.001, r=0.979)$ as expected. Moreover, we found that a Ki-67 $>4 \%$ was significantly associated with shorter survival in patients with ACC.

The Helsinki score that associates two Weiss criteria and the Ki-67 with a cut-off value of 8.5, can diagnose metastatic adrenocortical carcinoma with $100 \%$ sensitivity and $99.4 \%$ specificity [35]; it also is correlated to survival in patients with ACC [35]. In our series Weiss score $>5(p=0.01)$ and Helsinki score $>8(p=0.06)$ were significantly associated with lower overall survival, in the univariable analysis. In an attempt to find predictive and prognostic immunohistochemical markers of adrenocortical carcinoma, we proceed to investigate the expression of several markers including melan-A, synaptophysin, calretinin, inhibin-A and to correlate it with Ki-67, Weiss score, Helsinki score and overall survival [36].

Inhibin-A was positive in $95.5 \%$ of our cases and its correlation with Weiss score showed a trend towards significance. In consistent with our findings, Weissferdt et al. showed largely diffuse cytoplasmic reactivity of inhibin-A in $92.5 \%$ of cases of ACC [37].

Melan A (MART-1) mainly expressed in malignant melanomas, is also a widely used biomarker for adrenal cortical and other streoidogenic tissues with high specificity and sensitivity. We found positive melan-A expression in $75.5 \%$ of ACCs. In line, small series with 10 and 30 ACCs have shown positive reactivity to melan- $A$ in 60 and $97 \%$, respectively $[28,38]$. Melan-A expression was not related with stage, Ki-67, Weiss score, Helsinki score, in our cases.

Calretinin appears to be a stable marker for ACC and reported series show reactivity in $70-98 \%$ of cases $[37,39]$. In our series, nuclear and cytoplasmic staining was reported in $88.8 \%$ of cases. No correlation between calretinin and stage, Weiss score, Helsinki score and Ki-67 was observed.

We also found a positive synaptophysin staining in $88.6 \%$ of ACC cases, while a marginal positive association between Weiss score and the expression of synaptophysin was observed.

A positive correlation between vimentin expression and Weiss score was also established, according to our data. Previous study by Satelli et al., reported that vimentin-positive tumor cells were detected in $73.2 \%$ of ACC, while only $14.0 \%$ of adrenal cortical adenoma showed scattered vimentin-positive cells [40].

Vimentin was previously considered as a marker for epithelial-mesenchymal transition (EMT), however its active role in cancer cell dissemination has recently been recognized [41]. In a case report by Bulzico et al. on an aggressive metastatic ACC (Weiss-score of 9), the staining for vimentin showed an intense diffuse cytoplasmic immunohistochemical pattern [42]. The strong expression of mesenchymal proteins such as vimentin by tumoral cells could possibly lead to the acquisition of properties such as migration, invasive, and apoptosis resistance [42-44]. Of note, Sbiera et al. showed that in ACC, there is no indication of EMT as all adrenocortical tissues lacked expression of epithelial markers and exhibited closer similarity to mesenchymal tissues. However, higher SLUG expression (mesenchymal marker) was associated with clinical markers indicating aggressiveness [45].

Of interest, among the studied immunohistochemical markers, only melan-A expression was independently related with longer overall survival in the univariate analysis of our series. To note, lack of melan-A immunostaining has been associated with reduced disease-free and overall survival in melanomas [46]. Zlatibor et al. in a small series of ACC, reported that negative staining for inhibin A and synaptophysin was significantly associated with shorter survival, which was not confirmed in our study [28]. Among the main genes have been implicated as tumor drivers in sporadic ACC, is TP53 [47]. In the context of adult ACC the TP53 somatic mutations are presented in 16 and $21 \%$ according to two recent genome-wide studies $[48,49]$.

The majority of the studies [50, 51], albeit not all [52], have shown that $\mathrm{p} 53$ somatic mutations are prognostic factor and correlated with more advanced stages, with shorter disease free survival and poor outcome in ACCs.

In our IHC analysis, we found no relation of $\mathrm{p} 53$ overexpression with Weiss score, as well as disease stage. However, there was a strong relation between p53 overexpression and higher ki-67, as well as higher Helsinki score. More interestingly, in multivariable analysis overexpression of p53 was independent risk factor for shorter survival $(p=0.036)$, implying a higher risk of death by 21.29 times. In summary, our data reveal that expression of melan-A and lower expression of Ki-67 ( $\leq 4)$ were independently associated with longer OS time. In multivariable analysis, tumor volume, Weiss score $>5$ and overexpression of p53 were independent risk factors for shorter survival.

A limitation of our study is the small number of participants however adrenocortical carcinoma is a rare cancer. Moreover, the follow up period is not long enough, therefore in some cases the median survival time have not been reached yet. Finally, we did not include the inoperable adrenocortical carcinomas. 
Strength of our study is that the surgical team as well as the diagnostic pathologists did not change during the period of 20 years that the cases have been collected.

It is certain that a combination of markers for a more accurate differential diagnosis is still needed for the appropriate assessment of the adrenal tumors. The appropriate combination of clinical, histopathological and molecular parameters will improve in the future the prognostication of patients with ACC and will open the road for personalized management of these patients. The accurate diagnosis would probably protect the patients with a better outcome from an aggressive management after complete resection of the tumor with avoidance of heavy adjuvant treatment, such as mitotane and cytotoxic chemotherapy. It would also reveal the patients with an intermediate or poor outcome that need a more intensive follow up strategy and the use of more aggressive systemic adjuvant therapies.

Until then, the most important factors that determine long-term prognosis of ACC remain the disease stage at diagnosis, the Weiss score, and the Ki67 index. Immunohistochemical markers such as Melan A could serve as prognostic factor, however further studies are needed to prove its usefulness.

\section{Conflict of Interest}

The authors declare that they have no conflict of interest.

\section{References}

[1] Bilimoria KY, Shen WT, Elaraj D et al. Adrenocortical carcinoma in the United States: Treatment utilization and prognostic factors. Cancer 2008; 113: 3130-3136

[2] Kebebew E, Reiff E, Duh QY et al. Extent of disease at presentation and outcome for adrenocortical carcinoma: Have we made progress? World J Surg 2006; 30: 872-878

[3] Wanis KN, Kanthan R. Diagnostic and prognostic features in adrenocortical carcinoma: A single institution case series and review of the literature. World J Surg Oncol 2015; 13: 117

[4] Crona J, Beuschlein F. Adrenocortical carcinoma - towards genomics guided clinical care. Nat Rev Endocrinol 2019; 15: 548-560

[5] Ayala-Ramirez M, Jasim S, Feng L et al. Adrenocortical carcinoma: Clinical outcomes and prognosis of 330 patients at a tertiary care center. Eur J Endocrinol 2013; 169: 891-899

[6] Hofland J, Feelders RA, van der Wal R et al. Serum inhibin pro-alphaC is a tumor marker for adrenocortical carcinomas. Eur J Endocrinol 2012; 166: 281-289

[7] Allolio B, Fassnacht M. Clinical review: Adrenocortical carcinoma: Clinical update. J Clin Endocrinol Metab 2006; 91: 2027-2037

[8] Fassnacht M, Allolio B. Clinical management of adrenocortical carcinoma. Best Pract Res Clin Endocrinol Metab 2009; 23: 273-289

[9] Fassnacht M, Kroiss M, Allolio B. Update in adrenocortical carcinoma. J Clin Endocrinol Metab 2013; 98: 4551-4564

[10] Terzolo M, Stigliano A, Chiodini I et al. AME position statement on adrenal incidentaloma. Eur J Endocrinol 2011; 164: 851-870

[11] Lalli E. Adrenocortical development and cancer: focus on SF-1. J Mol Endocrinol 2010; 44: 301-307
[12] Duregon E, Cappellesso R, Maffeis $V$ et al. Validation of the prognostic role of the "Helsinki Score" in 225 cases of adrenocortical carcinoma. Hum Pathol 2017; 62: 1-7

[13] Jouinot A, Bertherat J. Management of endocrine disease: Adrenocortical carcinoma: Differentiating the good from the poor prognosis tumors. Eur J Endocrinol 2018; 178: R215-R230

[14] Berruti A, Baudin E, Gelderblom H et al. Adrenal cancer: ESMO clinical practice guidelines for diagnosis, treatment and follow-up. Ann Oncol 2012; 23: vii131-vii138

[15] Lughezzani G, Sun M, Perrotte P et al. The European network for the study of adrenal tumors staging system is prognostically superior to the international union against cancer-staging system: A North American validation. Eur J Cancer 2010; 46: 713-719

[16] Fassnacht M, Johanssen S, Fenske W et al. Improved survival in patients with stage II adrenocortical carcinoma followed up prospectively by specialized centers. J Clin Endocrinol Metab 2010; 95: 4925-4932

[17] Zografos GN, Vasiliadis G, Farfaras AN et al. Laparoscopic surgery for malignant adrenal tumors. JSLS 2009; 13: 196-202

[18] Alanee S, Dynda D, Holland B. Prevalence and prognostic value of lymph node dissection in treating adrenocortical carcinoma: A national experience. Anticancer Res 2015; 35: 5575-5579

[19] Gervasoni JE Jr., Sbayi S, Cady B. Role of lymphadenectomy in surgical treatment of solid tumors: an update on the clinical data. Ann Surg Oncol 2007; 14: 2443-2462

[20] Angelousi A, Kyriakopoulos G, Athanasouli F et al. The role of immunohistochemical markers for the diagnosis and prognosis of adrenocortical neoplasms. J Pers Med 2021; 11

[21] Kobel M, Piskorz AM, Lee S et al. Optimized p53 immunohistochemistry is an accurate predictor of TP53 mutation in ovarian carcinoma. J Pathol Clin Res 2016; 2: 247-258

[22] Wang YC, Lin RK, Tan YH et al. Wild-type p53 overexpression and its correlation with MDM2 and p14ARF alterations: an alternative pathway to non-small-cell lung cancer. J Clin Oncol 2005; 23: 154-164

[23] Angelousi A, Kassi E, Kaltsas GA et al. Current issues in the diagnosis and management of adrenocortical carcinomas. In: Feingold KR, Anawalt B, Boyce A et al. (Eds). Endotext. South Dartmouth (MA): MDText.com, Inc.; 2000

[24] Lam AK. Update on adrenal tumours in 2017 World Health Organization (WHO) of endocrine tumours. Endocr Pathol 2017; 28: 213-227

[25] Angelousi A, Kassi E, Kaltsas GA et al. Adrenocortical carcinoma. In: Feingold KR, Anawalt B, Boyce A et al. (Eds). Endotext. South Dartmouth (MA): MDText.com, Inc.; 2000

[26] Kahramangil B, Kose E, Remer EM et al. A modern assessment of cancer risk in adrenal incidentalomas: Analysis of 2219 patients. Ann Surg 2020. doi:10.1097/SLA.0000000000004048

[27] Kostiainen I, Hakaste L, Kejo P et al. Adrenocortical carcinoma: Presentation and outcome of a contemporary patient series. Endocrine 2019; 65: 166-174

[28] Zlatibor L, Paunovic I, Zivaljevic V et al. Prognostic significance of immunohistochemical markers in adrenocortical carcinoma. Acta Chir Belg 2020; 120: 23-29

[29] Nunes JM, Rodrigues E, Rios E et al. Adrenocortical carcinoma - a 25 years tertiary centre experience and short review of the literature. Endocrinol Metab Int J 2018; 6: 15-19

[30] Bedrose S, Daher M, Altameemi L et al. Adjuvant therapy in adrenocortical carcinoma: reflections and future directions. Cancers (Basel) 2020; 12

[31] Erickson LA. Challenges in surgical pathology of adrenocortical tumours. Histopathology 2018; 72: 82-96

[32] Morimoto R, Satoh F, Murakami O et al. Immunohistochemistry of a proliferation marker Ki67/MIB1 in adrenocortical carcinomas: Ki67/ MIB1 labeling index is a predictor for recurrence of adrenocortical carcinomas. Endocr J 2008; 55: 49-55 
[33] McNicol AM, Struthers AL, Nolan CE et al. Proliferation in adrenocortical tumors: correlation with clinical outcome and p53 status. Endocr Pathol 1997; 8: 29-36

[34] Stojadinovic A, Ghossein RA, Hoos A et al. Adrenocortical carcinoma: clinical, morphologic, and molecular characterization. J Clin Oncol 2002; 20: 941-950

[35] Pennanen M, Heiskanen I, Sane T et al. Helsinki score-a novel model for prediction of metastases in adrenocortical carcinomas. Hum Pathol 2015; 46: 404-410

[36] Sangoi AR, Fujiwara M, West RB et al. Immunohistochemical distinction of primary adrenal cortical lesions from metastatic clear cell renal cell carcinoma: A study of 248 cases. Am J Surg Pathol 2011; 35: 678-686

[37] Weissferdt A, Phan A, Suster S et al. Adrenocortical carcinoma: A comprehensive immunohistochemical study of 40 cases. Appl Immunohistochem Mol Morphol 2014; 22: 24-30

[38] Mondal SK, Dasgupta S, Jain P et al. Histopathological study of adrenocortical carcinoma with special reference to the Weiss system and TNM staging and the role of immunohistochemistry to differentiate it from renal cell carcinoma. J Cancer Res Ther 2013; 9: 436-441

[39] Beuschlein F, Weigel J, Saeger W et al. Major prognostic role of Ki67 in localized adrenocortical carcinoma after complete resection. J Clin Endocrinol Metab 2015; 100: 841-849

[40] Satelli A, Li S. Vimentin in cancer and its potential as a molecular target for cancer therapy. Cell Mol Life Sci 2011; 68: 3033-3046

[41] Perez-Sala D, Oeste CL, Martinez AE et al. Vimentin filament organization and stress sensing depend on its single cysteine residue and zinc binding. Nat Commun 2015; 6: 7287

[42] Bulzico D, Torres DC, Ferreira GM et al. A novel TP53 mutation associated with TWIST1 and SIP1 expression in an aggressive adrenocortical carcinoma. Endocr Pathol 2017; 28: 326-331
[43] Kalluri R, Weinberg RA. The basics of epithelial-mesenchymal transition. J Clin Invest 2009; 119: 1420-1428

[44] Zeisberg M, Neilson EG. Biomarkers for epithelial-mesenchymal transitions. J Clin Invest 2009; 119: 1429-1437

[45] Sbiera I, Kircher S, Altieri B et al. Epithelial and mesenchymal markers in adrenocortical tissues: How mesenchymal are adrenocortical tissues? Cancers (Basel) 2021; 13

[46] Berset M, Cerottini JP, Guggisberg D et al. Expression of Melan-A/ MART-1 antigen as a prognostic factor in primary cutaneous melanoma. Int J Cancer 2001; 95: 73-77

[47] Babinska A, Peksa R, Wisniewski P et al. Diagnostic and prognostic role of SF1, IGF2, Ki67, p53, adiponectin, and leptin receptors in human adrenal cortical tumors. J Surg Oncol 2017; 116: 427-433

[48] Assie G, Letouze E, Fassnacht $M$ et al. Integrated genomic characterization of adrenocortical carcinoma. Nat Genet 2014; 46: 607-612

[49] Zheng S, Cherniack AD, Dewal $N$ et al. Comprehensive pan-genomic characterization of adrenocortical carcinoma. Cancer Cell 2016; 30: 363

[50] Juhlin CC, Goh G, Healy JM et al. Whole-exome sequencing characterizes the landscape of somatic mutations and copy number alterations in adrenocortical carcinoma. J Clin Endocrinol Metab 2015; 100: E493-E502

[51] Lippert J, Appenzeller S, Liang R et al. Targeted molecular analysis in adrenocortical carcinomas: a strategy toward improved personalized prognostication. J Clin Endocrinol Metab 2018; 103: 4511-4523

[52] Waldmann J, Patsalis N, Fendrich V et al. Clinical impact of TP53 alterations in drenocortical carcinomas. Langenbecks Arch Surg 2012; 397: 209-216 